\title{
Para todos o para nadie. El debate sobre la Ley de Zonas Francas en el Congreso de la Nación Argentina *
}

\section{For Everyone or Nobody. The Free Zone's Law Debate in the Argentinian Parliament}

\section{Mauricio Moltó}

\author{
Mauricio Moltó es becario del Consejo Nacional \\ de Investigaciones Científicas y Técnicas en la \\ Universidad Nacional del Litoral, Argentina. \\ E-mail: mauriciomolto@gmail.com
}

\section{resumen}

Durante la década de 1990, la agenda económica argentina se vio traccionada por la idea de "volver al mundo". En paralelo con la deslegitimación de la intervención estatal, ganaron terreno discursos relacionados con la competitividad y la eficiencia. En este contexto, resurgió la idea de dotar al país de una normativa para la regulación de actividades en las zonas francas. En este sentido, interesa analizar el proceso mediante el cual la idea de las zonas francas como dispositivo de desarrollo económico retorna a la agenda pública argentina. Asimismo, observaremos las diferentes posiciones e intereses que se entrecruzaron durante el tratamiento de la Ley 24.331. La hipótesis es que la posibilidad de crear zonas francas funcionó como punto de equilibrio entre la demanda de políticas públicas económicas por parte de las provincias y la oferta de herramientas disponibles en manos del gobierno nacional, las cuales le permitían mantenerse dentro de los márgenes discursivos de la posición ideológica.

\section{summary}

During the 1990's, Argentinian economic agenda was influenced by the idea of "insert back the country in the world". In this way, simultaneously to the state action's discredit, ideas such as production efficiency and economic competitiveness were winning influence. In this context the idea of regulate the activities of free zones was reborn. Thus, this work aims to analyze the free zones' return to the Argentinian public agenda and the political positions which were confronted during the 24.331 law debate. The hypothesis is that the possibility of create free zones worked like an encounter point between the provinces' economic public policies demands and the National Government action possibilities in a neoliberal ideology context which dominated the Argentina's economics ideas during the 1990's.

\section{palabras clave}

zona franca / debate parlamentario / diseño de políticas públicas

\section{keywords}

free zones / parliamentary debate / public policy design 


\section{Introducción}

Durante la década de 1990, la agenda económica argentina estuvo traccionada por la idea de "volver al mundo". Esto, entre otras cosas, en términos prácticos, significaba la desregulación del comercio exterior y la habilitación al capital extranjero para hacerse cargo de la administración de las ex empresas públicas. En esa sintonía, en paralelo con la deslegitimación de la intervención estatal y la creciente injerencia de organismos internacionales en las decisiones del Estado nacional, ganaron terreno discursos relacionados con la competitividad y la eficiencia. En este contexto, resurge la idea de dotar al país de una normativa para la regulación de actividades industriales y comerciales en zonas francas (ZF). En consecuencia, el gobierno nacional envió al Congreso el proyecto de lo que poco tiempo después se convertiría en la Ley 24.331.

La existencia de una agenda de problemas públicos (Cobb y Elder, 1993) implica una relación entre gobierno y sociedad donde los actores de la sociedad civil logran que el gobierno seleccione o incorpore sus inquietudes, demandas o intereses entre las cuestiones a resolver. También, a la inversa, implica que el gobierno puede establecer relaciones con actores de la sociedad civil para construir el consenso necesario para llevar adelante sus intereses. Esta dinámica, puede pensarse, se desarrolla mediante relaciones entre actores sociales que forman parte de $-\mathrm{y}$ le dan forma a- un campo en el cual sus participantes intentan imponer su propia definición del problema público $\mathrm{y}$, a su vez, legitimar sus intereses materiales y simbólicos a través del Estado. Esto vale tanto para actores y grupos de la sociedad civil como para funcionarios de gobierno y coaliciones políticas, quienes operan como contraparte activa y mediante su participación intentan hacer valer su mirada y posición en torno a uno o más asuntos (Lorenc Valcarce, 2005).

En este sentido, el objetivo es analizar el proceso mediante el cual la idea de crear zonas francas retorna a la agenda pública argentina, así como las diferentes posiciones e intereses que se entrecruzaron durante el tratamiento de la Ley 24.331. Se trabaja a partir de la hipótesis de que la posibilidad de crear zonas francas funcionó como el punto de equilibrio entre la demanda de políticas públicas económicas por parte de las provincias y la oferta de herramientas disponibles en manos del gobierno nacional, las cuales le permitían mantenerse dentro de los márgenes discursivos de la posición ideológica dominante de la época.

A los fines de comprender los posicionamientos e intereses de los distintos actores implicados en el diseño de una política pública de zonas francas en Argentina, se analizaron los diarios de sesiones de las tres jornadas durante que se debatió el tema en el Congreso: 7 de diciembre de 1993, 15 de marzo de 1994 y 18 de mayo del mismo año. Se identificaron las posiciones de los legisladores frente a la Ley y se reconstruyeron las cinco categorías que mencionamos en el párrafo siguiente, las cuales dieron forma al marco de sentido del debate. A su vez, creemos que el análisis significa un aporte para comprender el modo en que se construyen las políticas públicas en el país, principalmente en lo que respecta a la relación que establecen el poder central y las provincias, así como entre los propios Estados subnacionales, cuyas relaciones, a su vez, están atravesadas por lógicas de cons- 
trucción de poder territorial y, también, hacia el interior de los partidos y/o alianzas parlamentarias.

El texto se divide en dos partes. En la primera, se realiza una caracterización general sobre las zonas francas en el país, sus antecedentes normativos y la llegada de la Ley al Congreso. En la segunda, se presentan los resultados del análisis de los diarios de sesiones agrupados en cinco categorías emergentes: 1) autonomías provinciales; 2) modelo económico; 3) relaciones de las ZF con el territorio; 4) economías regionales; y 5) Mercosur y países limítrofes.

\section{Zonas francas: aspectos conceptuales y normativos}

Una ZF es un dispositivo de comercio exterior cuyo objetivo es el desarrollo y la promoción de las capacidades exportadoras de una economía. Su versión moderna surgió durante la crisis del modo de producción fordista y su reemplazo por un modelo económico basado en las ideas de competitividad y descentralización, que propició la distribución de los eslabones de las cadenas productivas en distintos países, la declinación de los mercados internos y el aumento del comercio internacional. El caso paradigmático es el de la ZF ubicada en el aeropuerto de Shannon, en Irlanda, creada en el año 1959. Como modo de aprovechar el viraje del país desde la sustitución de importaciones hacia un modelo económico basado en la inserción exportadora de la economía, la ZF de Shannon operó como un elemento dinamizador del crecimiento económico y el desarrollo industrial del oeste irlandés durante la década de 1960 (Madoery, 1996).

Las ZF constituyen regímenes de excepción con beneficios impositivos especiales. Existen diferentes tipos de conceptos utilizados para describir las variantes de esta herramienta: zona franca comercial, zona empresarial especial, zona económica especial, zona de servicios financieros, zona de comercio exterior, maquiladoras, zonas de procesamiento de exportaciones (Madoery, 1996).

De acuerdo con información de la AFIP, en Argentina existen doce zonas francas en funcionamiento. La primera en entrar en operaciones fue la Zona Franca Justo Daract (San Luis), en el año 1997, mismo año en que comenzó a operar la Zona Franca de La Plata. La más reciente, de momento, es la Zona Franca Santafesina, que comenzó su actividad durante los primeros meses de 2018. Las demás son las siguientes: Bahía Blanca - Coronel Rosales (2011); Comodoro Rivadavia (2001); Concepción del Uruguay, en Entre Ríos (2008); Córdoba (1998); Cruz Alta, en Tucumán (1998); General Güemes, en Salta (2001); General Pico, en La Pampa (1999); Luján de Cuyo, en Mendoza (1999); y Puerto Iguazú (2002). A esta lista es preciso sumar el régimen aduanero especial de Tierra del Fuego, del año 1972.

Amendolara (2009) señala que el Ministerio de Economía de la Nación habilitó, además, reglamentos de funcionamiento presentados por distintas provincias para las zonas francas de Clorinda, Formosa (1997); Corrientes (1997); Chamical y Felipe Varela, en La Rioja (1997); Frías, Santiago del Estero (1996); Perico y La Puna, Jujuy (1996); Río Gallegos y Caleta Olivia (1995); San Juan (1996); Sierra Grande, Río Negro (1996); Tinogasta, Catamarca (1998); y Zapala, Neuquén (1996). Más allá del estado en que se encuentre la implementación en cada uno 
de estos casos, los datos indican que las provincias han realizado un avance para sumarse a la propuesta del Estado nacional, o bien que en algún momento tuvieron la intención hacerlo.

La norma que regula el funcionamiento de las ZF en Argentina es la Ley Nacional 24.331 y su última complementaria, la Ley 25.379. A la legislación mencionada, se suman la serie de artículos que van desde el 590 hasta el 599 del Código Aduanero (Ley 22.415). En su artículo cuarto, la Ley 24.331 estipula que "las zonas francas tendrán como objetivo impulsar el comercio y la actividad industrial exportadora, facilitando que el aumento de la eficiencia y la disminución de los costos asociados a las actividades que se desarrollan en ellas, se extiendan a la inversión y al empleo" y, convergentemente con la política comercial nacional, contribuyan "al crecimiento y a la competitividad de la economía y a incorporarse plenamente en el proceso de integración regional". Para el cumplimiento de estos objetivos, el Estado nacional invita a las provincias a su adhesión y, de esta forma, posibilita la creación de una ZF en cada una de las provincias adherentes, más otras cuatro sujetas a decisión del gobierno nacional. La Ley establece un entramado complejo de relaciones entre distintas jurisdicciones que, desde un punto de vista jurídico, no siempre son de fácil caracterización. Para ver en detalle estas relaciones puede consultarse a Moscariello (2013).

Una de las relaciones más discutidas durante el debate en el Congreso fue la que establece el artículo 2 de la norma entre el Poder Ejecutivo Nacional (PEN) y las provincias. Según este artículo, es el PEN quien está autorizado para la creación de ZF en las provincias y también se reserva, a través del Ministerio de Economía, Obras y Servicios Públicos, la autoridad de aplicación de la Ley. Las provincias, por su parte, deben crear una comisión de evaluación y selección en el marco del Poder Ejecutivo Provincial (PEP) que estudie las propuestas para la concesión de la ZF, así como también elaborar un reglamento que deberá ser remitido para su aprobación a la autoridad de aplicación.

Esta dinámica se encuentra en línea con las transformaciones llevadas adelante en la administración pública nacional a principios de la década de 1990, cuando el Estado central renunció a funcionar como prestador directo de bienes y servicios económicos para adoptar un papel de regulador del mercado -en el caso de las empresas privatizadas- $\mathrm{y}$ de planificador $\mathrm{y}$ formulador de políticas y lineamientos generales de gestión en relación con los Estados subnacionales (Oszlak, 2003). De todas formas, al reservarse el PEN discrecionalidad para la creación de cuatro ZF, mantiene una carta que eventualmente puede ser usada para apuntalar la política de ZF si las provincias llegaran a no implementarlas, algo que pasó en muchos casos. Asimismo, esa discrecionalidad puede ser direccionada en favor del gobierno nacional en caso de utilizar una eventual creación de ZF como moneda de cambio en la negociación con alguna de las provincias.

La historia en materia de legislación sobre ZF en el país se remonta hasta el año 1907. En ese año, la Ley Nacional 5.142 habilitó la creación de dos ZF, una de ellas en la Provincia de Buenos Aires, en la ciudad de La Plata, y la otra en la Provincia de Santa Fe, sin especificar ubicación. También, en el año 1910, me- 
diante la Ley 8.092, se habilitó la creación de una ZF en la ciudad entrerriana de Concepción del Uruguay.

Hubo que esperar el paso de casi la totalidad del siglo XX para ver avances al respecto, ya que fue recién en 1991 cuando el Decreto Nacional 1.688 -luego derogado y reemplazado por el Decreto 1.788- estableció la ubicación específica de la Zona Franca de La Plata y limitó su esfera de acción a la actividad comercial. La creación de la Zona Franca de La Plata desató gran expectativa en las provincias, las cuales intentaron obtener una ley que las favoreciera en esa misma línea. Santa Fe y Entre Ríos aprovecharon, como lo hizo Buenos Aires, la legislación de principio de siglo nunca implementada. El decreto 1.935 reglamenta la Zona Franca de Concepción del Uruguay en base a la Ley 8.092; mientras que el Decreto 2.034, en base a la Ley 5.147, crea una ZF en la ciudad de Villa Constitución, en el extremo sureste de Santa Fe. De todas formas, ninguna de las dos ZF se puso en funcionamiento de inmediato, sino que pasaron 16 y 26 años respectivamente hasta que comenzaron a operar. Lo más importante, sin embargo, es que el Decreto 1.688 creó, bajo la órbita del Ministerio de Economía y Obras y Servicios Públicos, una comisión técnica a los efectos de evaluar y proponer al PEN lineamientos para un proyecto para legislar sobre Zonas Francas.

Las motivaciones del PEN para avanzar hacia una reglamentación sobre ZF fueron varias. El mensaje que acompañó el anteproyecto del Ejecutivo, en sintonía con el clima ideológico de la época, señalaba que el ordenamiento de las actividades de las ZF se inscribía en el contexto de una política económica que apuntaba a corregir las "distorsiones históricas" responsables de la crisis y estancamiento en el país que, por medio de "un conjunto de decisiones de política oportunamente implementadas pretendieron y pretenden restituir su rol a los mercados buscando simultáneamente insertar a la Argentina en el mundo" (Honorable Cámara de Diputados, 1993: 3901). De esta forma, mediante este dispositivo, el PEN consideraba estar dando un paso más hacia la remoción del sesgo "antiexportador" que afectaba la economía nacional. En este sentido, existía la expectativa de que las ZF pudieran aumentar la eficiencia económica del sistema, el volumen de exportaciones y la recepción de inversión extranjera directa.

Lo cierto es que para el momento en que el Gobierno de Menem decidió tomar la iniciativa en torno a las ZF, el tema ya se encontraba en agenda y había al menos 16 proyectos para creación de ZF en distintos lugares del país. Esta situación es ilustrativa de lo que señalan Spiller y Tommasi (2011) con relación al bajo nivel de cooperación existente para el diseño de políticas públicas en el Congreso Nacional. Evidencia, asimismo, una disputa por parte de algunas provincias para conseguir materializar en sus propios territorios, antes que en el resto, un dispositivo que por entonces se creía que iba a operar como dinamizador de la actividad económica.

Las intervenciones de Diputados y Senadores hicieron alusiones a esos proyectos de autoría propia o de sus colegas, y en muchos casos se atribuyeron haber sido los primeros en pedir la instalación de una ZF para su provincia. Otras veces, denunciaron que su proyecto no había sido considerado en la redacción del pro- 
yecto del PEN y/o durante el trabajo en las comisiones. Todos ellos basaron sus argumentos en las necesidades que atravesaban sus provincias, los kilómetros de frontera compartidos con países limítrofes y/o la ubicación estratégica para la nación de tal o cual punto de la geografía provincial. Lo cierto es que desde fines de la década de 1980, y hasta que el Ejecutivo envió su propuesta, ingresaron al Congreso una cantidad de proyectos que generaron una situación que bien describe con ironía la apreciación realizada por el Diputado Raúl Vicchi (UCR Mendoza) durante el debate en la Cámara Baja:

El problema que se daba para sancionar esta ley data del momento en que una gran cantidad de diputados presentaron proyectos en el mismo sentido, por supuesto todos pensando que estas zonas francas encuentran su lugar ideal de asentamiento en sus propias provincias. Es así como nos encontramos en un callejón sin salida, ya que no íbamos a poder decir u opinar ni siquiera racionalmente cuál iba a ser el espacio físico donde pudiéramos asentar la primera de esas zonas francas (...) (Honorable Cámara de Diputados, 1993: 29).

Por esta razón, el PEN ideó una solución que se mostró eficiente para no generar conflictos dentro de su bloque legislativo y que le evitó quedar en posición de árbitro y pagar el consecuente costo político de elegir una provincia en vez de otras: el proyecto de Ley habilitó a todas las provincias a crear una ZF en su territorio, siempre y cuando suscribieran a la Ley sancionada por el Congreso y encausaran su proyecto de ZF de acuerdo con la Ley marco. Este punto fue uno de los aspectos técnicos más problemáticos durante el debate, debido a que habilitó la posibilidad de crear $28 \mathrm{ZF}$ :

[Diputado Victorio Bisciotti, UCR Buenos Aires] En general, los países, salvo algunas excepciones, sólo han establecido una o dos zonas francas. Estos son regímenes de excepción y no se puede abusar de ellos (...). Si leemos los fundamentos de la creación de las zonas francas, se podría llegar a la conclusión de que es racional hacerlo, pero crear tantas zonas francas como provincias existen, es irracional, porque se diluyen los efectos deseados y no se tienen en cuenta las consecuencias que originan en el universo económico del país. Esto es grave (Honorable Cámara de Diputados, 1993: 3909).

Otra de las motivaciones que alentaron al Ejecutivo a tomar cartas en el asunto fue la cuestión regional. Hacia mediados de la década de 1990, existían ZF en Brasil, Bolivia, Chile, Paraguay y Uruguay. Además, el 1 de enero de 1995, terminado el período de transición, entraba en vigencia plenamente el Mercosur. A raíz de eso, se temía que, luego de esa fecha, fuese más difícil compatibilizar la legislación sobre el tema.

El tratamiento duró un poco más de un año desde que el proyecto "Zonas francas: reglamentación de actividades en las mismas", firmado por Carlos Menem y Domingo Cavallo, ingresó al Congreso de la Nación el 19 de mayo de 1993 y fue remitido a la Cámara de Diputados. Una vez allí fue girado a cuatro comisiones: 
Economía; Comercio; Presupuesto y Hacienda; y Economías y Desarrollo Regional. Luego del trabajo en comisiones, el 10 de noviembre ingresó al orden del día para ser tratado en el recinto. El 7 de diciembre fue aprobado con modificaciones y pasó al Senado. En la Cámara de Senadores fue revisado por tres comisiones: Presupuesto y Hacienda; Industria; y Comercio. El 15 de marzo de 1994 fue aprobado con modificaciones y volvió a Diputados. Dos meses más tarde, el 18 de mayo, la Cámara Baja dio sanción a la Ley, la cual fue promulgada por el PEN luego de realizar un veto parcial. Su versión final fue publicada el 17 de junio en el Boletín Oficial.

\section{Debate de la Ley 24.331}

Las deliberaciones en el Congreso de la Nación se realizaron sobre el piso de un importante consenso en torno a la necesidad de regular la habilitación y funcionamiento de las ZF. Como se dijo anteriormente, la temática ya estaba instalada en la agenda parlamentaria, principalmente a causa de los proyectos presentados por legisladores de varias provincias. En este sentido, en el debate no se reflejaron fuertes posiciones encontradas entre los diferentes partidos o bloques parlamentarios.

En la sesión del 7 de diciembre de 1993, el Diputado Héctor Álvarez (PJ Misiones) presentó una moción para el tratamiento sobre tablas del proyecto del PEN. La votación tuvo un nivel relativamente bajo de participación -51,8\%-, pero un nivel de adhesión que fue de casi $88 \%$. Si bien el Bloque Justicialista se encolumnó detrás de la propuesta del Ejecutivo, no hubo un bloque netamente opositor al proyecto. La UCR, así como los bloques menores sumaron Diputados tanto por la positiva como por la negativa. Cabe destacar que entre los opositores y las abstenciones se encontraron todos diputados de Buenos Aires, Capital Federal, Entre Ríos, Santa Fe y Córdoba (Década votada, 2018). Se trataba de todas las provincias ubicadas en la pampa húmeda, las cuales ya contaban con legislación habilitante para crear una ZF en su territorio, a excepción de Córdoba y Capital Federal. Por el contrario, el proyecto generó alta expectativa en las provincias del interior, cuyos legisladores fueron los más activos durante el debate. Algunos gobernadores hicieron llegar su voz mediante cartas e, incluso, el entonces Gobernador de Santa Cruz, Néstor Kirchner, estuvo presente durante la sesión del 18 de mayo en Diputados, hecho que, en parte, explica por qué todos los Diputados santacruceños tomaron la palabra ese día. En el Senado, el proyecto también fue tratado sobre tablas y el 29 de diciembre de 1993 se votó una moción de preferencia para que sea tratado en la primera sesión del siguiente año.

Los votos opositores al proyecto fueron pocos, pero no sucedió lo mismo con las críticas. En la lectura de los diarios de sesiones en Diputados y Senadores, se identificaron tres tipos de posturas: quienes apoyaron el proyecto del PEN con entusiasmo y sin críticas, bajo la interpretación de que se trataba de un nuevo paso hacia la integración del país con el mundo; los adherentes críticos, quienes plantearon objeciones muy duras pero que, ante la falta de una alternativa supera- 
dora con consenso, votaron positivamente; y los opositores, quienes, además de plantear duras críticas, no apoyaron el proyecto.

A continuación, se realiza un repaso por cinco categorías emergentes durante el análisis de los diarios de sesiones, en un intento por mostrar las diferencias entre las tres posturas mencionadas: 1) las autonomías provinciales; 2) el modelo económico; 3) las relaciones de las ZF con el territorio; 4) las economías regionales; y 5) el Mercosur y las relaciones con los países limítrofes.

\section{Autonomías provinciales}

El procedimiento estipulado para la creación de las ZF en el proyecto del PEN fue objeto de críticas por parte de los legisladores. Mientras los más entusiastas se limitaron a señalar que la iniciativa ponía a todas las provincias en pie de igualdad - principalmente a causa de que Buenos Aires, Santa Fe y Entre Ríos ya podían crear sus ZF y el resto de las provincias no-, los más críticos interpretaron que la redacción de la Ley vulneraba las autonomías provinciales. Como se mencionó anteriormente, el artículo 2 fue bastante resistido y se le introdujeron modificaciones significativas durante la discusión en el Congreso. De todos modos, dichas modificaciones fueron descartadas en su totalidad por el PEN, el cual dejó solo una breve referencia a la situación de las provincias limítrofes. Originalmente, el texto del artículo $2^{\circ}$ decía:

Facúltese al Poder Ejecutivo Nacional para crear en el territorio de cada provincia una zona franca, incluyéndose las ya existentes a los efectos de este cómputo, pudiendo con carácter de excepción crear adicionalmente no más de cuatro (4) en todo el territorio nacional, a ser ubicadas en aquellas provincias o regiones geográficas, que en pos su situación socioeconómica crítica justifiquen la necesidad de este instrumento de excepción (Honorable Cámara de Diputados, 1993: 3992).

El punto de discusión fue que, más allá de que la Ley habilita a cada provincia para crear una ZF, la última palabra al respecto la tiene el PEN. En ese sentido, se señaló que la redacción del texto daba demasiadas atribuciones al gobierno nacional. Tampoco fue muy bien recibida la posibilidad de que la Ley otorgue potestad a este último para crear cuatro ZF independientes de las que pudieran crear las provincias. Ante esta situación, las críticas se extendieron hacia el requisito de adherir a la Ley nacional que plantea el artículo 3. Algunos legisladores argumentaron que la potestad para crear ZF debería ser del Congreso de la Nación, bajo el amparo en lo dispuesto por el código aduanero en su artículo 591, que sostiene que las ZF deben ser creadas por ley:

[Diputado Victorio Bisciotti, UCR Buenos Aires] El artículo $3^{\circ}$ determina que se condiciona la creación de las zonas francas en las provincias a la firma por parte de ellas de un convenio de adhesión cuyo contenido no figura especificado. Si la zona franca se debe crear por ley de la Nación, ¿para qué se determina la realización de un convenio que no conocemos? ¿Responde al interés del país? Aparentemente la intención es crear zonas 
francas donde se haga mejor lobby o donde mejor se hayan alineado a las directivas políticas o a lo que marque el ministro de Economía (Honorable Cámara de Diputados, 1993: 3910).

En el comentario anterior se puede ver, además, una manifiesta desconfianza hacia el gobierno en relación con los usos políticos que hubiera podido hacer con las ZF.

De acuerdo con la Ley, el Ministerio de Economía y Obras y Servicios Públicos funcionaba como autoridad de aplicación de la Ley, mientras que las provincias eran las encargadas de proponer la ubicación de la ZF y, una vez aprobada por el Ministerio, debían crear un organismo mixto que funcionara como comité de vigilancia. Por esta razón, entre los desacuerdos fueron frecuentes aquellos comentarios que señalaron la concentración de poder en manos del entonces Ministro Cavallo:

[Senador José Genoud, UCR Mendoza] Adviértase ahora todas las facultades que están concentradas en la persona del señor Ministro de Economía. En primer lugar, es quien en definitiva resuelve el lugar del establecimiento de las zonas francas. Me pregunto por qué tiene que decidir el Ministro de Economía Cavallo respecto del lugar en que conviene radicar una zona franca, por ejemplo, en mi provincia. Esto está quebrando un principio de autonomía elemental que señalamos a modo de impugnación (...) (Honorable Cámara de Senadores, 1994: 5943).

Otro punto que suscitó controversias fue el artículo 31, que estableció que los gobiernos provinciales no podrán disponer exenciones impositivas distintas de las previstas en la Ley nacional. Al respecto, los legisladores alineados con el proyecto del Ejecutivo consideraron que este aspecto daba un piso de igualdad a todas las provincias y fue defendido incluso por algunos legisladores de la oposición, quienes veían el contenido del mencionado artículo como una forma de evitar la competencia entre las diferentes Zonas Francas provinciales. En este sentido se manifestó el entonces Senador de la UCR por Capital Federal Fernando De la Rúa:

Tampoco me parece mal que se prohíba a las provincias disponer exenciones de impuestos locales, y voy a decir por qué. No porque considere que la Nación pueda avasallar los derechos de las provincias; no hay un problema de avasallamiento, sino una razón para prohibir este tipo de exenciones. Se trata de querer mantener la igualdad de posibilidades, de oportunidades, para todas las provincias. De lo contrario, se generaría una guerra de competencia entre las provincias para ver cuál va a dar mayores beneficios para atraer hacia ella posibles inversores (Honorable Cámara de Senadores, 1994: 5957).

Sin embargo, desde las provincias más alejadas de la capital, algunas voces fundamentaban la necesidad de poder disponer de exenciones. Se argumentó que las fuertes asimetrías regionales del país provocaban que algunas provincias fueran 
más competitivas que otras. En este sentido, el artículo 36 reforzaba las ventajas de las provincias centrales, dotadas de mayor infraestructura y en una posición geográfica privilegiada que implicaba, por ejemplo, menores costos de transporte:

[Diputado Raúl Vicchi, UCR Mendoza] La forma cerrada con la que se ha manejado la gente del Ministerio de Economía con este tema no ha posibilitado que tengamos un debate amplio y sincero para dirimir dónde hace falta una cosa y dónde hace falta otra, ya que a lo mejor este régimen impositivo puede ser perfectamente implementado en una zona franca ubicada en La Plata (...) [pero] el régimen impositivo a aplicar en una zona franca del sur de nuestro país o a una perdida en la Puna debe ser diferente al que está en vigencia para todo el país a efectos de que tengan posibilidad de competir (Honorable Cámara de Diputados, 1994: 689-690).

\section{El modelo económico}

El debate sobre las ZF, por tratarse de un tema con un sesgo técnico importante, transcurrió por los márgenes de la agenda política. Esto se vio acentuado por la inserción en un contexto donde se estaban discutiendo asuntos de vital trascendencia, como los preparativos para la Reforma Constitucional de 1994. Sin embargo, los problemas del nuevo modelo económico, que ya se comenzaban a sentir en algunas provincias, se hicieron presentes en el debate parlamentario de la Ley 24.331.

Los entusiastas del proyecto interpretaron la creación de ZF como un paso más hacia la consolidación de una economía nacional integrada al mundo. Consideraban que se trataba de una iniciativa coherente con la política económica del gobierno nacional, que realizaba un avance para solucionar los problemas históricos del país producto de los vicios del estatismo y la ineficiencia ocasionada por una economía cerrada. El comentario del Senador Fernando Cabana (PJ Jujuy) ilustra bien el sentido en que se interpretó la herramienta por parte de los participantes más entusiastas del debate:

Cuatro años y medio es poco tiempo para la vida humana, ¿qué es, entonces, para un país? Es un instante, señor presidente, y en ese instante el país ha experimentado una transformación revolucionaria. De ese país sumido en las tinieblas del Tercer Mundo, hemos pasado a ser el líder de América Latina y el interlocutor igualitario de los países más poderosos (...). Ahora se trata de este proyecto de zonas francas que tiende a facilitar inversiones, fundamentalmente en industrias que exportan. Los eternos pedigüeños dirán que esto es insuficiente y que llevará mucho tiempo. Pero, ¿qué empresa sólida no requiere, para obtener frutos, esfuerzo y tiempo? (Honorable Cámara de Senadores, 1994: 5962).

Del comentario anterior se desprende el apoyo hacia la totalidad de la política económica del Gobierno de Menem y, por extensión, a la Ley sobre ZF. Producto de esta actitud, la reflexión acerca de la utilidad del instrumento o los problemas de implementación que demandaría quedaron en un segundo plano y primó el alineamiento automático con el PEN. 
A causa de la superación de la crisis hiperinflacionaria, existía un sólido consenso en torno a la convertibilidad. Sin embargo, tanto en Diputados como en Senadores no faltaron voces críticas sobre los efectos perniciosos de la sobrevaluación del peso y la apertura económica para las economías regionales, principalmente por parte de aquellos legisladores de provincias limítrofes con Paraguay y Brasil. Esta situación ocasionó una postura cercana a la resignación por parte de estos legisladores, quienes vieron en el debate sobre las ZF una oportunidad para poner en agenda los problemas económicos de sus provincias. Manifestaron su apoyo a la iniciativa del PEN con la esperanza de que pudiera servir para hacer frente a las dificultades por las que atravesaban sus provincias, aunque reconocieron también que no se trataba de una herramienta ideal o una solución integral a una problemática que se manifestaba de manera compleja. Durante estas intervenciones, se encontraron críticas al gobierno nacional por la falta de políticas económicas para mitigar los efectos negativos de la apertura de mercados y la convertibilidad. Al respecto, es ilustrativo el comentario del Diputado Marcelo B. Muñoz (UCR Chaco):

No hacen falta solamente zonas francas, sino una zona aduanera especial, como también una política coherente de desarrollo de las economías regionales (...). De este gobierno podríamos rescatar la estabilidad, ya que por lo menos hasta ahora tenemos una política financiera. Hay grietas en el sistema, pero por lo menos hay estabilidad. Sin embargo, nadie puede dudar de que no tenemos una verdadera política de desarrollo. Desregular no significa renunciar a planificar. Y lo que estoy temiendo es que estemos mimetizando la desregulación olvidándonos de la planificación (Honorable Cámara de Diputados, 1994: 671).

Los más críticos, por su parte, señalaron el proyecto como una iniciativa improvisada que respondía a negociaciones políticas entre el gobierno nacional y algunos gobernadores, pero que no daba respuesta a problemáticas de fondo en materia de política económica. En este sentido, se manifestó, por ejemplo, el ya citado Diputado Bisciotti:

(...) es un remedio de excepción y para el cual hay que partir de un análisis global de la política industrial, económica y comercial del país. Lo que sucede es que en la realidad, salvo el plan de convertibilidad, la Argentina no cuenta con una política adecuada en ninguna de las materias que acabo de señalar. Por eso se está decidiendo ahora este popurrí de zonas francas que responden, en definitiva, a los lobbies que operan (Honorable Cámara de Diputados, 1993: 3910).

Las consecuencias negativas del modelo económico ya se percibían en algunas regiones del país. Sin embargo, la fortaleza del consenso en torno a los éxitos mostrados por el plan de convertibilidad en materia de estabilización monetaria y la pérdida de legitimidad del Estado y la regulación de la economía obligaban a pensar soluciones dentro de la matriz neoliberal. Por esa razón, ante algunas de las 
desventajas evidentes del modelo y la falta de alternativas, algunos legisladores se esforzaban en mantener el equilibrio. Intentaban transmitir el malestar económico de sus provincias, pero con mucho cuidado de no quedar asociados con un discurso estatista o demasiado crítico del mainstream, que les restara atractivo político hacia el interior de sus espacios políticos y frente a la opinión pública.

\section{Relaciones con el territorio aduanero general}

Las ZF constituyen un régimen económico de excepción con actividades en una pequeña área delimitada y separada del territorio aduanero general (TAG), donde se controla el ingreso y egreso de personas y mercaderías. La Ley 24.331 se remite al artículo 590 del Código Aduanero, en el cual se establece que un

área franca es un ámbito dentro del cual la mercadería no está sometida al control habitual del servicio aduanero y su introducción y extracción no están gravadas con el pago de tributos, salvo las tasas retributivas de servicios que pudieren establecerse, ni alcanzadas por prohibiciones de carácter económico.

Complementariamente, el artículo 6 de la Ley 24.331 brinda la posibilidad de desarrollar actividades de almacenamiento, comercio, servicios e industriales con fines de exportación, que permiten solo el ingreso de bienes industriales de capital que no registren antecedentes de fabricación en el TAG.

El tratamiento fiscal especial de las ZF persigue el objetivo de hacer más competitivas las actividades desarrolladas dentro de su perímetro y, de este modo, potenciar la oferta exportadora del país. Los beneficios para el TAG se esperan como producto de los posibles encadenamientos que generen las actividades de las ZF con las del área geográfica circundante y, también, de las nuevas fuentes de trabajo que generen las operaciones en la ZF. En este sentido, aun con algunos reparos o escepticismo en torno al efecto multiplicador, las ZF como instrumento fueron bien recibidas y el debate apenas rozó el tratamiento de la pertinencia del dispositivo para promover el desarrollo regional y la creación de empleo. Incluso, algunos vieron en la reducción de costos para las actividades industriales oportunidades para desarrollar nichos de innovación y desarrollo productivo:

[Diputado Horacio Macedo, PJ Jujuy] Me parece oportuno aclarar que es posible encontrar múltiples actividades y procesos, tanto para el mercado interno argentino como para la exportación, con la utilización de materias primas, insumos, mano de obra y empresas argentinas. Es decir que esto aparece como una excelente oportunidad para eliminar o disminuir el llamado costo argentino (Honorable Cámara de Diputados, 1994: 685).

En esta línea, hubo acuerdo en cuanto a la potencialidad de la herramienta, pero algunos legisladores plantearon que, dada la situación de sus provincias, una pequeña área delimitada no era suficiente para hacer frente a la competencia generada por los productos provenientes de países limítrofes. En este sentido, como una forma de reconocimiento implícito de las ventajas del régimen de excepción 
propuesto por el PEN, solicitaron la ampliación del área geográfica y la creación territorios aduaneros especiales (TAE). Luego del tratamiento en el Senado, el proyecto volvió a la Cámara Baja con modificaciones. La más significativa fue el agregado de cuatro artículos en un tercer título que establecían y regulaban TAE en la Puna -que abarcaba parte de las provincias de Catamarca, Salta y Jujuy- y en la Patagonia Austral, que comprendía parte de las provincias de Santa Cruz, Chubut y el municipio de Sierra Grande en Río Negro. El nuevo Título III de la propuesta del Senado pasó sin modificaciones por Diputados, pero fue vetado por el PEN.

Otro punto de debate fue la potestad que el artículo 9 de la Ley 24.331 otorgaba al PEN para habilitar operaciones de comercio al por menor cuando "las circunstancias lo aconsejen". Al respecto, hubo una fuerte crítica por parte de algunos legisladores que acusaron al gobierno nacional de pretender crear 28 Free Shops o Shoppings. Sin embargo, algunos legisladores de provincias limítrofes plantearon la posibilidad de que en las ZF pudiera existir venta minorista para contrarrestar la competencia ejercida por los precios más bajos existentes al otro lado de la frontera. Finalmente, las ventas al por menor se habilitaron solo para ciudades fronterizas en provincias con una densidad poblacional menor a 2 habitantes por kilómetro cuadrado, lo cual en la práctica solo favoreció a Santa Cruz.

Por otra parte, acorde al clima de época, el proyecto del PEN pretendía instaurar un régimen de flexibilización laboral hacia el interior de las ZF. Por entonces, la flexibilización laboral estaba en agenda y algunos legisladores denunciaron el peligro que significaba para los derechos laborales de los trabajadores de todo el país permitir la flexibilización en las ZF. El intento del gobierno nacional por avanzar en este sentido no prosperó, ya que se estableció que en las ZF regiría la misma legislación laboral que en el TAG. Se trataba del mismo caso que la legislación medioambiental y financiera, sobre la cual se alertó ante posibles actividades de lavado de dinero: gran parte de la desconfianza existente hacia las ZF se fundaba en la posibilidad de hechos de corrupción, lo cual habilitaría una porción del territorio desregulada:

[Senador Luis León, UCR Chaco] Desde ya solicito que quede como una aspiración el hecho de que todos los señores concesionarios que pretendan tener el manejo de estas zonas sean mirados con lente muy argentino, muy moral, porque hay en el país una intoxicación de deformaciones que posiblemente se inserten también en este tema. Si las grandes industrias pueden producir algunos hechos "truchos" en el manejo de la propia importación y exportación, qué podría pasar si algunos funcionarios o intereses privados entran a manejar áreas importantes con un cierto grado de libertad, como el que tendrán los concesionarios que posean el control de estas zonas (Honorable Cámara de Senadores, 1994: 5956).

El comentario anterior, de alguna manera, refleja la sensación de desconfianza que existía entre algunos legisladores respecto de la forma en que se estaban llevando adelante las reformas estatales y las privatizaciones, que, como señala 
Thwaites Rey (2003), en muchos casos se hicieron incluso a contramano de las recomendaciones de los organismos internacionales.

\section{Las economías regionales}

Durante el tratamiento de la Ley se manifestaron fuertes expectativas sobre la utilidad de la futura norma para impulsar el desarrollo de las economías regionales. Las economías regionales se definen en oposición a las actividades económicas pampeanas. Mientras estas últimas se encuentran conectadas a los flujos de comercio internacional y tienen una importante inserción exportadora, las economías regionales, de desarrollo más tardío, nacieron como proveedoras de los grandes centros de consumo del mercado interno (Rofman, 1993).

A causa de la apertura económica y la falta de competitividad de algunos sectores de la economía nacional, se generaron serios problemas de empleo en todo el país. La falta de competitividad de la producción se explica por varias razones, las cuales se vieron catalizadas por la sobrevaluación del peso (Buscaglia, 2001). No obstante, esta problemática, a entender de los legisladores, se hizo sentir mucho más fuera de la pampa húmeda:

[Diputado Alejandro Nieva, UCR Jujuy] Lo que quiere decir es que no existe en el pueblo de la provincia de Buenos Aires la misma expectativa [sobre la creación de ZF] que tienen los habitantes del interior, sencillamente porque no sufren el grado de angustia y desesperanza que se evidencia en las distintas provincias (Honorable Cámara de Diputados, 1994: 682).

Los legisladores de las provincias extrapampeanas coincidieron en señalar los problemas de las economías del interior en zonas más despobladas y alejadas de Buenos Aires. En la misma línea que el discurso sobre las "provincias pobres" analizado por Vaca y Cao (2006) durante el tratamiento de las leyes de Coparticipación Federal de Impuestos y de Promoción Industrial en la década de 1970, los legisladores vieron en la iniciativa del PEN en torno a las ZF una oportunidad o un avance para solucionar la crisis económica de sus provincias:

[Senador Guillermo Snopek, PJ Jujuy] (...) pero sí debo decir que tanto a los hombres de esa zona [noreste argentino] como a los del noroeste nos beneficiará la aplicación de estos mecanismos que posibiliten la concreción de una esperanza a través de un proceso de reactivación mediante el cual se generen polos de desarrollo con sentido regional y proyectando su producción no sólo para el tradicional mercado de Buenos Aires — para el que fueron diseñados en el siglo pasado nuestros ferrocarriles - sino también para lograr competir en otros mercados como los de la cuenca del Pacífico (Honorable Cámara de Senadores, 1994: 5936).

Así y todo, en muchos casos, más que con optimismo, la propuesta del Ejecutivo fue recibida con resignación y cautela:

[Diputado Alberto Parada, PJ Chubut] Sabemos que el proyecto que vamos a sancionar no es la panacea, como algunos creen. Pero desde un 
principio dejamos en claro que tanto este proyecto como otros que había en danza no significan la solución total al problema que sufren las economías regionales. Por ello fue que tuvimos especial cuidado en no crear falsas expectativas. Simplemente estamos intentando dar algunas salidas a la grave situación que vive el interior del país (Honorable Cámara de Diputados, 1994: 663).

Se esperaba que las ZF pudieran propiciar una articulación de estrategias en base a los recursos disponibles en el territorio y una inserción internacional de la producción que tuviera como contraparte el desarrollo de encadenamientos de actividades en las provincias, expectativa cercana a la idea de desarrollo local (Albuquerque, 2004; Boisier, 2001; Vázquez Barquero, 2000). La otra posibilidad -menos contemplada- era que las ZF pudieran generar dinámicas socioeconómicas de enclave (Falero, 2015) con escaso efecto multiplicador en el territorio.

\section{El Mercosur y las relaciones con los países limitrofes}

El PEN presentó el proyecto de ZF como parte de una serie de medidas tendientes a disminuir el sesgo "antiexportador". Sin embargo, las temáticas o inquietudes relacionadas con el comercio internacional no pasaron de referencias al saldo negativo de la balanza comercial y a la carencia de un modelo de desarrollo por fuera de la apertura de la economía y el plan de convertibilidad. De esta forma, se ocupó una proporción de tiempo relativamente baja para discutir o expresar expectativas sobre la radicación de inversiones y la inserción de la economía en los circuitos globales del capitalismo que podrían posibilitar las ZF. En cambio, sí hubo un número importante de referencias al Mercosur y a los países limítrofes.

Estas referencias no apuntaron tanto al sentido estratégico de la herramienta en un marco de integración regional, sino que fueron vistas como un dispositivo defensivo que debía ser habilitado antes de que sea tarde. En la mirada de los participantes del debate, todos los países limítrofes ya contaban con ZF o instrumentos similares cerca de la frontera con Argentina y su funcionamiento perjudicaba las economías regionales:

[Diputado Pedro Figueroa, Movimiento Popular Jujeño] Lo cierto es que no podemos caer en la ingenuidad de seguir discutiendo el instrumento cuando Uruguay ya las está instalando enfrente de nuestras provincias mesopotámicas, cuando el presidente boliviano ya anunció que en las fronteras con nuestro país instalarán zonas francas, y cuando Chile tiene una enorme presencia por el norte con Iquique y por el sur con Punta Arena a través de instrumentos parecidos (Honorable Cámara de Diputados, 1993: 3912).

Sin embargo, el principal argumento para dotar al país de legislación sobre ZF probablemente estuvo dado por las discusiones que se desarrollaban en el seno del Mercosur, las cuales fueron saldadas con la Resolución 8 del año 1994 del Consejo del Mercado Común. Por medio de esta norma, se estableció que las exportaciones realizadas desde ZF de los países del bloque hacia otros miembros debían pagar el 
arancel externo común (Madoery, 1996). Más importante aún, sin embargo, es el contenido del artículo 5 de la Resolución, el cual habilita a funcionar en los países del Mercosur solo a las ZF existentes hasta ese momento o a crearse en base a legislación vigente en cada país (Mercosur, 1994). La Ley 24.331 es de junio de 1994; la Resolución 8 del Consejo del Mercado Común, de diciembre del mismo año.

Entre 1991 y 1994, crecieron de forma significativa las importaciones medidas como porcentaje del PBI, las cuales pasaron de representar un 4,4\% en 1991 a un $7,1 \%$ en 1994 . No obstante, no ocurrió mismo con las exportaciones, cuya evolución fue levemente decreciente, y pasó de representar 6,3\% en 1991 a 5,6\% en 1994 (Madoery, 1996). En esos mismos años, se dio un fuerte crecimiento del intercambio con los países del Mercosur, principalmente con Brasil. El comercio intrabloque pasó de representar el $16,5 \%$ de las exportaciones y $21,8 \%$ de las importaciones en 1991, hasta el orden de 30,3\% de las exportaciones y $26,6 \%$ de las importaciones en 1994 (Mellado, 1998). De cualquier modo, más allá de los datos concretos, el proceso de integración regional fue señalado por las provincias limítrofes como un aspecto perjudicial para sus economías, a causa de la sobrevaluación del peso, que fue percibida como un factor que impactó de lleno sobre la competitividad. Por esa razón, a pesar de ver con buenos ojos la posibilidad de instrumentar ZF, todas las provincias limítrofes pedían, además, consideraciones por fuera de lo dispuesto por el proyecto de ley: ser declaradas TAE, como en los casos mencionados anteriormente; ser declarada ZF en su totalidad, como el pedido que realizó el radicalismo de Misiones para su provincia; o recibir alguna de las ZF extra que podía crear el Ejecutivo:

[Senador Juan R. Aguirre Lanari, Partido Liberal de Corrientes] La provincia de Corrientes es un ejemplo muy singular, ya que linda con tres países hermanos en la frontera viva, quizá la más extensa del país. Reclamamos desde ya una consideración de las autoridades nacionales encargadas de dictaminar en los beneficios y zonas a crearse para poder afrontar el difícil desafío de la integración. Con estos auspicios, ayudando a la creación de estos polos de desarrollo que impulsen cambios económicos y sociales positivos para nuestros pueblos, podremos afrontar la competitividad con los países hermanos en beneficio de nuestras economías regionales (Honorable Cámara de Senadores, 1994: 5951).

\section{Conclusión}

La intención del Estado nacional de instrumentar una política de ZF como herramienta para la promoción de exportaciones era coherente con la situación económica global y nacional. Sin embargo, se vio cómo su diseño estuvo atravesado por lógicas ajenas a la política industrial y comercial, lo cual implicó realizar concesiones que le restaron coherencia y efectividad. Como señalaron algunos Diputados participantes del debate, el número de ZF que habilitó la legislación fue excesivo y muchas de ellas, pensaron, probablemente caerían, producto de la competencia. Era una solución que, de todos modos, se mostraba muy en sintonía con el clima de época. Además, el sentido que adquirió la iniciativa para los legislado- 
res fue problemático, ya que se interpretó una herramienta de política económica de corte liberal en clave keynesiana, con la esperanza de que las Zonas Francas pudieran servir para generar empleo y reactivar el mercado interno.

Se vio cómo la dinámica y las tensiones internas del sistema federal argentino restaron coherencia y racionalidad técnica al diseño de esta política pública. La posibilidad de crear ZF funcionó como una de las medidas posibles para la promoción del desarrollo económico y el comercio exterior en el marco de la hegemonía neoliberal, pero dada la gran cantidad de proyectos presentados en este sentido en el Congreso entre fines de la década de 1980 y principios de los años noventa, el PEN resolvió habilitar a todas las provincias a crear sus propias ZF. Por este motivo, puede decirse que la decisión del Gobierno Nacional fue también una decisión de política doméstica; una forma de evitar situaciones que pudieran restarle apoyo político de aquellos gobernadores que no hubieran quedado satisfechos con la ubicación de las ZF decididas por el Ejecutivo.

Al poner a jugar con las mismas reglas a regiones del país con distintas dotaciones de recursos humanos, infraestructura y posibilidad de conexión con mercados internacionales, el propio diseño de la iniciativa conspiró en contra de la idea de desarrollo regional presente entre sus objetivos. Tal vez el mayor problema, no obstante, se haya dado en la implementación de la política.

Richard Elmore (1993: 258-259) señaló que "el surgimiento de la implementación como objeto de estudio del análisis de políticas está estrechamente relacionado con el descubrimiento, por parte de los analistas, de que las decisiones no producen efectos inmediatos". En este sentido, llama la atención cómo una medida con fuerte consenso tomó tanto tiempo en instrumentarse. También, es notable cómo provincias limítrofes cuyos legisladores habían sido participantes entusiastas del debate no habilitaron su ZF y, por el contrario, sí lo hicieron en términos relativamente rápidos provincias mediterráneas como San Luis, La Pampa, Córdoba y Tucumán. A su vez, son llamativos los casos de Entre Ríos y Santa $\mathrm{Fe}$, provincias a las que, a pesar de no depender originalmente de la Ley 24.331, les tomó 16 y 26 años respectivamente armar su ZF. Por esta razón, se cree que el análisis de la implementación de la Ley 24.331 abre una puerta interesante para indagar sobre las relaciones y capacidad de acción coordinada para el desarrollo de políticas públicas entre el Estado nacional, las provincias y los municipios. En esta línea, entonces, se espera poder avanzar de aquí en adelante.

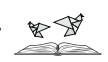

\section{Referencias}

* El contenido de este trabajo forma parte de la tesis doctoral "El Estado como solución y como problema. Actores públicos y privados en el proceso de implementación de la Zona Franca Argentina en la ciudad de Villa Constitución entre los años 1992 y 2018", realizada en el marco del Doctorado en Ciencias Sociales de la Facultad de Ciencias Sociales de la Universidad de Buenos Aires.

\section{Bibliografía}

Albuquerque, F. (2004). El enfoque del desarrollo económico local. Buenos Aires, Argentina: OIT. Disponible en: http://www.flacsoandes.edu.ec/sites/default/files/agora/files/1251776298.area 
enfoque_del_0.pdf

Amendolara, M. (2009). Estudio comparativo de las zonas francas en Argentina y España. Buenos Aires, Argentina: AFIP. Disponible en: http://www.afip.gob.ar/educaciontributaria/BibliotecaDigital/ documentos/C06.pdf

Boisier, S. (2001). Desarrollo local: ¿de qué estamos hablando? En O. Madoery y A. Vázquez Barquero (Eds.), Transformaciones globales, Instituciones y Políticas de desarrollo local Rosario, Argentina: Homo Sapiens. Disponible en: http://www.flacsoandes.edu.ec/web/imagesFTP/1245948918. Desarrollo_Local_De_que_estamos_hablando_2_.pdf

Buscaglia, A. (2001). La economía argentina a fines del siglo XX. En M. Lascano (Comp.), La economía argentina hoy. Buenos Aires, Argentina: Ateneo.

Cobb, R. y Elder, C. (1993). Formación de la agenda. El caso de la política de ancianos. En L. F. Aguilar Villanueva (Comp.), Problemas públicos y agenda de gobierno. México D. F., México: Fondo de Cultura Económica.

Elmore, R (1993). Diseño retrospectivo: la investigación de la implementación y las decisiones políticas. En L. F. Aguilar Villanueva (Comp.), La implementación de las políticas. México D. F., México: Fondo de Cultura Económica.

Falero, A. (2015). La expansión de la economía de enclaves en América Latina y la ficción del desarrollo: siguiendo una vieja discusión en nuevos moldes. Revista Mexicana de Ciencias Agrícolas, Vol. 1, Instituto Nacional de Investigaciones Forestales, Agrícolas y Pecuarias, Estado de México, México, 145- 157. Disponible en: http://www.redalyc.org/pdf/2631/263139243020.pdf

Honorable Cámara de Diputados de la Nación (1993). Diario de sesiones, $30^{\circ}$ reunión, continuación de la $4^{\circ}$ sesión especial, 12 de diciembre.

Honorable Cámara de Diputados de la Nación (1994). Diario de sesiones, $3^{\circ}$ reunión, continuación de la $1^{\circ}$ reunión ordinaria, 18 de mayo.

Honorable Cámara de Senadores de la Nación (1994). Diario de sesiones, $75^{\circ}$ reunión, $4^{\circ}$ sesión extraordinaria, 15 de marzo.

Lorenc Valcarce, F. (2005). La sociología de los problemas públicos. Una perspectiva crítica para el estudio de las relaciones entre la sociedad y la política. Nómadas, Nro. 12 (julio- diciembre), Universidad Complutense de Madrid, España. Disponible en: http://www.redalyc.org/html/181/18153295010/

Madoery, O. (1996). Zonas francas. Desarrollo regional y exportaciones. Buenos Aires, Argentina: Ad Hoc.

Mellado, N. B. (1998). Zonas Francas y MERCOSUR: la Zona Franca de La Plata. La Plata, Argentina: Ed. Barsa. Disponible en: http://sedici.unlp.edu.ar/handle/10915/59820

Mercosur (1994). Resolución $N^{o} 8$. Montevideo, Uruguay: Consejo del Mercado Común. Disponible en: http://www.loa.org.ar/legNormaDetalle.aspx?id=751

Moscariello, A. (2013). Zonas francas: análisis desde el derecho administrativo. El Derecho. Serie Administrativo, 9/10/2013, p. 5.

Oszlak, O. (2003). El mito del Estado mínimo: una década de reforma estatal en Argentina. Desarrollo Económico, Vol. 42, № 168. Enero-Marzo.

Rofman, A. (1993). Las economías regionales. Un proceso de decadencia estructural. En P. Bustos (Comp.), Más allá de la estabilidad, pp. 161-189. Buenos Aires, Argentina: Fundación Friedrich Ebert. Spiller, P. y Tommasi M. (2011). Un país sin rumbo. ¿Cómo se hacen las políticas públicas en Argentina?. En C. Scartascini et al. (Eds.), El juego político en América Latina, BID. Disponible en: https://publications.iadb.org/bitstream/handle/11319/332/El\%20juego\%20pol\%C3\%ADtico\%20 en $\% 20 \mathrm{Am} \% \mathrm{C} 3 \%$ A9rica $\% 20$ Latina $\% 3 \mathrm{~A} \% 20 \% \mathrm{C} 2 \%$ BFC $\%$ C3\%B3mo $\% 20$ se $\% 20$ deciden $\% 201$ as $\% 20$ pol $\%$ C3\%ADticas $\% 20$ p $\%$ C3\%BAblicas $\% 3 \mathrm{~F}$.pdf?sequence $=1$

Thwaites Rey, M. (2003). La (des)ilusión privatista. El experimento neoliberal en Argentina. Buenos Aires, Argentina: Libros del Rojas- EUDEBA.

Vaca, J. y Cao H. (2006). Discurso y políticas públicas. Las provincias “pobres” en la aprobación de los regímenes de promoción industrial y coparticipación federal de impuestos. Documento de trabajo del Centro de Investigaciones en Administración Pública, Facultad de Ciencias Económicas, Universidad de Buenos Aires, Argentina. 
Vázquez Barquero, A. (2000). Desarrollo económico local y descentralización: aproximación a un marco conceptual. Santiago de Chile, Chile: CEPAL. Disponible en: http://repositorio.cepal.org/ bitstream/handle/11362/31392/S00020088_es.pdf

\section{Fuentes}

Década votada (2018) recuperado de: http://www.decadavotada.com.ar/ (02 de octubre de 2018).

Decreto No 1688, Boletín Oficial de la República Argentina, 11 de septiembre de 1991 http://servicios. infoleg.gob.ar

Decreto No 1788, Boletín Oficial de la República Argentina, 01 de septiembre de 1993 http://servicios. infoleg.gob.ar

Decreto No 1935, Boletín Oficial de la República Argentina, 21 de octubre de 1992 http://servicios. infoleg.gob.ar

Decreto No 2034, Boletín Oficial de la República Argentina, 06 de noviembre de 1992. http://servicios. infoleg.gob.ar

Ley No 5142, Boletín Oficial de la República Argentina, Buenos Aires, 23 de septiembre de 1907 http:// servicios.infoleg.gob.ar

Ley $\mathrm{N}^{\circ}$ 8092, Boletín Oficial de la República Argentina, Buenos Aires, 28 de octubre de 1910 http:// servicios.infoleg.gob.ar

Ley N 22 415, "Código Aduanero", Boletín Oficial de la República Argentina, Buenos Aires, 23 de marzo de $1981 \mathrm{http}: / /$ servicios.infoleg.gob.ar

Ley N ${ }^{\circ} 24$ 331, "Zonas Francas”, Boletín Oficial de la República Argentina, Buenos Aires, 17 de junio de 1994, http://servicios.infoleg.gob.ar

Ley № 25 379, Boletín Oficial de la República Argentina, Buenos Aires, 03 de enero de 2001 http:// servicios.infoleg.gob.ar

Recibido: 11/03/2020. Aceptado: 20/06/2020.

Mauricio Moltó, "Para todos o para nadie. El debate sobre la Ley de Zonas Francas en el Congreso de la Nación Argentina". Revista Temas y Debates. ISSN 1666-0714, año 24, número 40, julio-diciembre 2020, pp. 13-31. 